\title{
A rare cause of left upper quadrant abdominal pain
}

\author{
Francesca Fiorentino • Andrea Bribani · \\ Alessandro Rosselli
}

Received: 8 April 2010/Accepted: 1 July 2010/Published online: 19 August 2010

(C) SIMI 2010

\section{Case presentation}

Dr. Fiorentino: A 44-year-old black woman from Cuba was admitted to the Emergency Department (ED) because of stabbing abdominal pain in the left upper quadrant, which had begun suddenly after she finished working. During the week prior to admission, she had no fever, no symptoms or signs of gastroenteritis, no dysuria, and no change in bowel habit. The patient said that she had the same symptoms 2 years prior to this episode after an airplane flight. On that occasion, she was admitted to a hospital, but she did not remember the diagnosis and no records were available. The patient also had no recollection of previous diagnosis of hemoglobinopathies, was not aware of previous health problems or diseases and had no recollection of childhood hospitalization.

On admission to the ED, she presented symptomatic with mild tenderness in the left upper quadrant of the abdomen. The vital signs were: pulse 93 beats/min, respiratory rate (RR) 16 breaths/min, blood pressure (BP) $130 / 85 \mathrm{mmHg}$, body temperature $36.7^{\circ} \mathrm{C}$. The finger stick glucose was $91 \mathrm{mg} \%$. The 12-lead ECG showed a sinus rhythm with a normal QRS shape.

Physical examination revealed a soft abdomen that was mildly tender in the left upper quadrant. There were no pulsating masses, no splenomegaly, no asymmetrical peripheral pulses nor detectable bruits. The liver was slightly enlarged on palpation. The remainder of the examination was unremarkable.

F. Fiorentino $(\bowtie) \cdot$ A. Bribani · A. Rosselli

U.O. Medicina d'Urgenza, Ospedale S. Maria Annunziata,

ASL 10, Via dell'Antella, 50100 Florence, Italy

e-mail: francescafiorentino1@alice.it
The results of routine laboratory findings, including $\beta$-HCG, ALT, AST, pancreatic amylase, bilirubin, creatinine, coagulation parameters were within normal range, except for a mild anemia $(\mathrm{Hb}=11.2 \mathrm{~g} / \mathrm{dl}$; $\mathrm{Htc}=33.4 \%$; $\mathrm{VCM}=80.5 \mathrm{fl})$, mild LDH $(903 \mathrm{U} / \mathrm{L})$ and PCR increase (3.29 mg/dl) [1, 2].

The ultrasound (US) abdominal examination [3] revealed an abnormal and inhomogeneous splenic echostructure because of multiple lesions, with the characteristics of ischemic infarctions and scarring (Fig. 1, see white arrows); there were no other pathological findings in the liver, kidneys, pancreas, abdominal aorta and pelvic organs.

Acute splenic infarctions appear as more or less echogenic lesions, without Doppler signal (Fig. 2; see white arrows), with variable dimensions, that extend to the surface. Their characteristic pyramidal shape allows us to distinguish splenic infarction from abscesses or tumoral infiltration. The chronic relapsing infarction is typical of sickle cell anemia. In this case, the spleen has multiple echogenic or hypoechoic focal lesions that led to a progressive splenic capsule retraction because of fibrous scars. During recovery there is an increase in echogenicity and the retraction of the infarcted area. The complications (20\% of all cases) are [4]: gradual colliquation (degeneration of splenic tissue to a liquid state after a necrotic process), sub-capsular bleedings, pseudoaneurysms or arterio-venous fistulae and hemoperitoneum. The possible evolutions are:

1. Full recovery,

2. Focal inhomogeneity in the infarcted area,

3. Colliquation with bleeding and pseudocyst formation,

4. Colliquation with impending rupture,

5. Peritoneal effusion. 


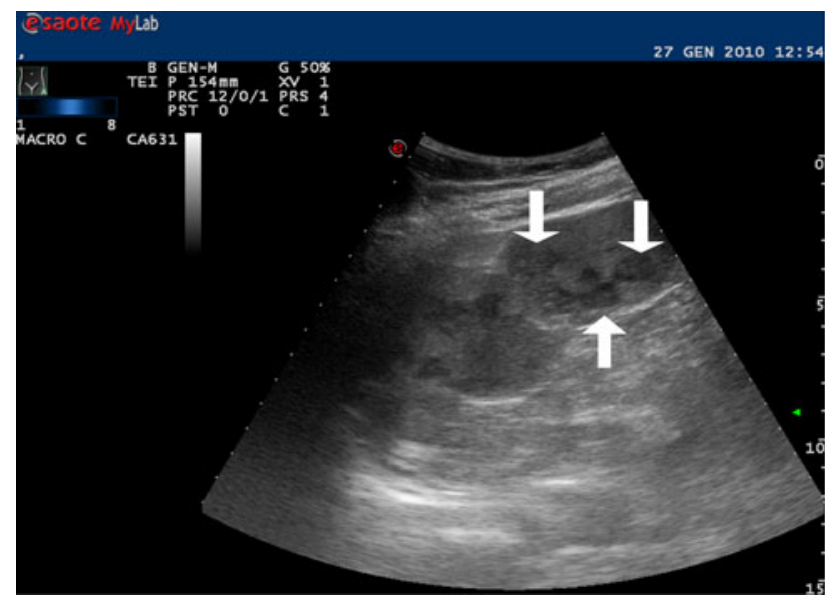

Fig. 1 Acute splenic infarctions appear as more or less echogenic lesions at ultrasound examination (arrows)

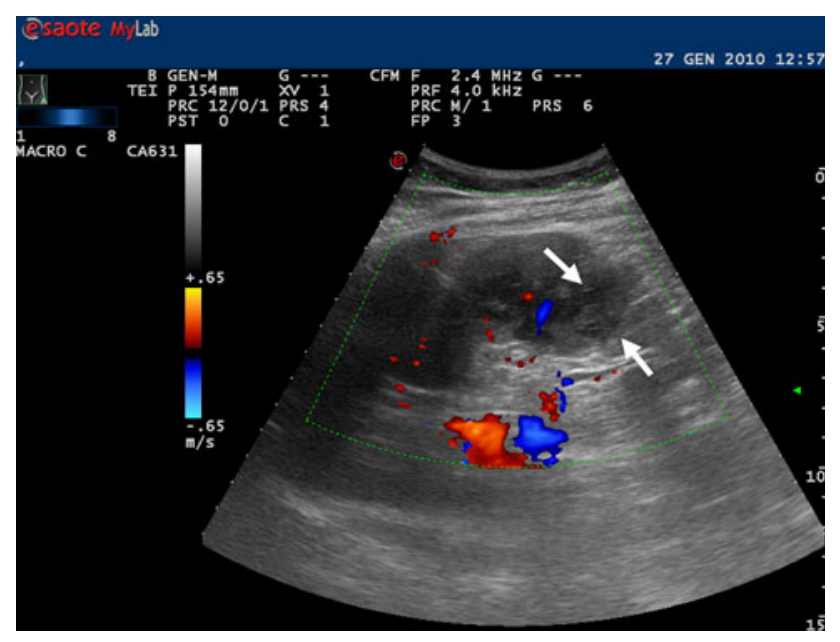

Fig. 2 Acute splenic ischemic infarctions do not have color-Doppler signal (arrows)

Our patient's clinical situation improved in a few days after bed rest, nasal oxygen and analgesia (paracetamol and codeine).

\section{Differential diagnosis}

Dr. Fiorentino, Dr. Bribani: Acute abdominal pain prevalent in the left upper abdominal quadrant could be caused by:

1. Splenic capsule acute stretching due to infectious diseases (mononucleosis, typhoid, etc), splenic abscess, malaria, chronic myeloid leukemia, splenic rupture,

2. Splenic infarction: past health history is usually positive for blood disorders (hemoglobinopathies, myeloproliferative disorders),

3. Splenic artery aneurism ready to rupture: in this case the patient usually has a recent pregnancy, and the pain is dull but with continual worsening, till the final clinical manifestation of hemoperitoneum and hemorrhagic shock,

4. Acute pancreatitis or caudal pancreatic pseudocyst, expanding or complicated by an infection,

5. Cancer of the left colic flexure, left kidney or adrenal glands cancer with superimposed necrosis, hemorrhage or infection,

6. Extra abdominal pathology: myocardial infarction, left lung lower lobe pneumonia.

The diagnostic hypothesis in this case was based on a few elements: the patient's race and past clinical history, the characteristic of abdominal pain onset, the site of the abdominal tenderness, the clues of hemolytic anemia in the laboratory tests, and the splenic anomalies on the ultrasound examination.

\section{Preliminary diagnosis}

Dr. Fiorentino: We made a preliminary diagnostic hypothesis of sickle cell disease on the basis of the following clinical elements: the patient's hometown, her black race, the characteristics of the abdominal pain, similar symptoms having occurred in the past after an airplane trip, the laboratory signs of hemolytic anemia, the ultrasonic splenic parenchymal inhomogeneities.

\section{Further investigations}

Dr. Bribani: The peripheral blood smear showed red cell anisocytosis (unequal-sized red blood cells), some target cells and a few dacrocytes (teardrop-shaped red blood cells). The haptoglobin was lower than normal $(2 \mathrm{mg} / \mathrm{dl})$ and there was reticulocytosis $\left(0.14 \times 10^{6} / \mu \mathrm{l}\right.$; normal range 0.018-0.114). Hemoglobin electrophoresis, the diagnostic test, revealed the presence of $\mathrm{HbS}(48.3 \%), \mathrm{HbC} 42.2 \%$, $\mathrm{HbF} 0.8 \%$, HbA2 3.7\% (normal range 2-3.3). A splenic US study with intravenous contrast Sonovue $(2.4 \mathrm{ml})$ confirmed the presence of relapsing infarctions (white arrows) and scarring (black arrows, Fig. 3). The spleen is easily studied by means of this technique because the contrast medium is eliminated very slowly: the splenic artery begins to opacify in 12-14 s after intravenous injection of contrast medium, then the whole parenchyma remains enhanced for 5-6 min.

\section{Discussion and conclusion}

Dr. Rosselli: In USA, almost 5,000,000 patients present to the ED because of abdominal pain (5-10\% of all ED visits). The diagnosis is not made in $30 \%$ of cases [5, 6]. 


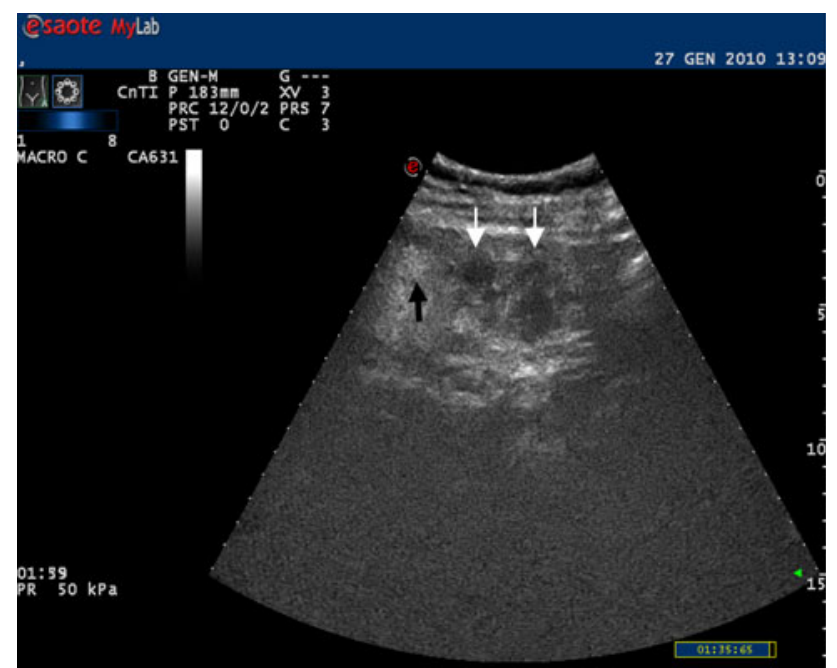

Fig. 3 Splenic infarctions (white arrows) and scarring (black arrows) revealed by the ultrasound study with intravenous contrast Sonovue

We illustrate one case of medical and not surgical abdominal pain due to a rare cause. The sickle cell syndromes are caused by a mutation in the $\beta$-globin gene that changes the sixth aminoacid from glutamic acid to valine. Several sickle syndromes occur as the result of inheritance of $\mathrm{HbS}$ from one parent and another hemoglobinopathy, such as $\beta$ thalassemia or $\mathrm{HbC}\left(\alpha 2 \beta 2^{6}\right.$ Glu $\rightarrow$ Lys $)$, from the other parent. Sickle cell syndromes are characterized by their clinical heterogeneity. Only patients who do not have true sickle disease may remain virtually asymptomatic into or even through adult life, while others suffer repeated crisis requiring hospitalization from early childhood. Hemoglobin SC disease, one of the more common variants of sickle cell anemia, is frequently marked by lesser degrees of hemolytic anemia and a greater propensity for the development of retinopathy and aseptic necrosis of bone [7]. Sickle cell disease patients typically present to the ED because of complications, most commonly venoocclusive painful crisis (musculoskeletal pain, hand-foot syndrome, acute chest pain syndrome, stroke, priapism), but also hematologic crisis (splenic sequestration, aplastic crisis, haemolytic crisis), infections (pneumonia, meningitis, sepsis, osteomyelitis, and urinary tract infections. While acute splenic sequestration and splenic infarction are commonly observed in infants and young children with sickle cell anemia, they are rarely experienced by adult hemoglobin $\mathrm{S}$ homozygotes because the recurrent splenic infarction that takes place during childhood is typically followed by scarring, atrophy and splenic fibrosis. Splenic infarction does remain relatively common in adults with the other sickle hemoglobinopathies, as in our case.

In this report, we describe a Central American woman of African origin, affected by hemoglobin SC disease, who developed abdominal pain due to splenic infarction without a clear trigger event, such as a history of recent air travel or exposure to altitude or use of any type of drugs.

This is a case of nonsurgical abdominal pain, caused by a rare genetic disorder, the initial diagnosis being made in the ED by means of non invasive and low cost examinations (laboratory tests and US). In particular, we avoided performance of an abdominal computed tomography (CT scan) in a still fertile woman.

Making the first diagnosis of a genetic, rare disorder in the ED may be possible; it is essential to first consider the possibility.

\section{Conflict of interest None.}

\section{References}

1. Rossato M et al (2009) Splenic infarction: a rare cause of acute abdominal pain presenting in an older patient with primary antiphospholipid antibodies syndrome. Intern Emerg Med 4(6):531-533

2. Koduri PR, Nathan S (2006) Acute splenic sequestration crisis in adults with hemoglobin S-C disease: a report of nine cases. Ann Hematol 85(4):239-243

3. Madani $\mathrm{G}$ et al (2007) The radiological manifestations of sickle cell disease. Clin Radiol 62(6):528-538

4. Tsaras G et al (2009) Complications associated with sickle cell trait: a brief narrative review. Am J Med 122(6):507-512

5. Koduri PR et al (2001) Hemoglobin SC disease revisited; clinical study of 106 adults. Am J Hematol 68(4):298-300

6. American College of Emergency Physicians (2000) Clinical policy: critical issue for the initial evaluation and management of patients presenting with a chief complaint of nontraumatic acute abdominal pain. Ann Emerg Med 36:406-415

7. Moll S et al (1997) Hemoglobin SC disease. Am J Hematol 54(4):313 